\title{
Tuning Riboflavin Derivatives for Photodynamic Inactivation of Pathogens
}

\author{
Ljiljana Fruk ( $\nabla$ If389@cam.ac.uk) \\ University of Cambridge \\ Leander Crocker \\ University of Cambridge \\ Ju Hyun Lee \\ University of Cambridge \\ Suraj Mital \\ University of Cambridge \\ Gabrielle Mills \\ University of Cambridge \\ Sina Schack \\ University of Cambridge \\ Andrea Bistrovic-Popov \\ University of Cambridge \\ Christoph Franck \\ University of Cambridge \\ loanna Mela \\ University of Cambridge \\ Clemens Kaminski \\ University of Cambridge \\ Graham Christie \\ University of Cambridge
}

\section{Research Article}

\section{Keywords:}

Posted Date: February 16th, 2022

DOI: https://doi.org/10.21203/rs.3.rs-1111998/v2

License: (1) This work is licensed under a Creative Commons Attribution 4.0 International License. Read Full License 


\section{Abstract}

The development of effective pathogen reduction strategies is required due to the rise in antibiotic-resistant bacteria and zoonotic viral pandemics. Photodynamic inactivation (PDI) of bacteria and viruses is a potent reduction strategy that bypasses typical resistance mechanisms. Naturally occurring riboflavin has been widely used in PDI applications due to efficient light-induced reactive oxygen species (ROS) release. By rational design of its core structure to alter (photo)physical properties, we obtained derivatives capable of outperforming riboflavin's visible light-iinduced PDI against $E$. coli and a SARS-CoV-2 surrogate, revealing functional group dependency for each pathogen. Bacterial PDI was influenced mainly by guanidino substitution, whereas viral PDI increased through bromination of the flavin. These observations were related to enhanced uptake and ROSspecific nucleic acid cleavage mechanisms. Trends in the derivatives' toxicity towards human fibroblast cells were also investigated to assess viable therapeutic derivatives and help guide further design of PDI agents to combat pathogenic organisms.

\section{Introduction}

Riboflavin and its derivatives, flavin mononucleotide (FMN) and flavin adenine dinucleotide (FAD) are organic cofactors found within enzymes involved in numerous biochemical pathways. ${ }^{1,2}$ Due to their rich redox chemistry and ability to mediate a wide range of both oxidative and reductive organic transformations, flavincontaining enzymes are prominently used as biocatalysts. ${ }^{3,4}$ Flavins are also involved in the regulation of photochemical pathways due to strong blue light absorption, which results in the generation of highly oxidising excited states that can elicit biological signalling events or responses. ${ }^{5-8}$ This photoexcitation process has been exploited in a number of photocatalytic applications, whereby modification of the flavin chromophore can enable the formation of high potential oxidative and reductive intermediates to afford useful synthetic methodology. ${ }^{9-}$ 11

Another application in which photoexcitation is utilised to generate reactive intermediates is within photodynamic inactivation (PDI) of pathogens. In this technique, a photosensitiser (PS) such as riboflavin, is employed to generate a burst of reactive oxygen species (ROS) in the vicinity of a particular pathogen in order to cause irreversible damage that leads to inactivation. ${ }^{12,13}$ Due to the rapid onset and non-specificity of PSinduced ROS release, conventional mechanisms of pathogen resistance through specific efflux pumps or detoxification pathways can be avoided. ${ }^{14}$ Therefore, PDI of pathogens is a versatile and effective strategy that has the potential to help combat increasing antibiotic-resistant bacteria strains, ${ }^{14,15}$ and ongoing zoonotic viral pandemics. ${ }^{16}$

Mechanistically, PDI of a pathogen is initiated by excitation of a PS with a suitable wavelength of light ( $\left.h v_{\text {ex }}\right)$ which generates an excited singlet state $\left(\mathrm{S}_{1}\right)$. This excited state can either fluoresce ( $\mathrm{hv}$ em $)$ back to its ground state $\left(\mathrm{S}_{0}\right)$ or undergo intersystem crossing (ISC) to an excited triplet state $\left(\mathrm{T}_{1}\right)$, typically characterised by a longer lifetime (Figure 1). This excited triplet state PS can interact with substrate such as an amino acid residue or nucleobase to form an oxidised substrate and reduced PS that subsequently reduces oxygen to superoxide $\left(\mathrm{O}_{2}{ }^{--}\right)$(Type I reaction, Figure 1). ${ }^{17}$ Superoxide is produced under oxidative stress naturally and is an important factor of cell signalling pathways. Both prokaryotic and eukaryotic cells contain superoxide dismutase (SOD) enzymes in order to mitigate superoxide's cytotoxic effects. ${ }^{17}$ Other types of ROS can be formed in Type I 
reactions such as $\mathrm{H}_{2} \mathrm{O}_{2}$ and hydroxyl radicals $\left({ }^{\circ} \mathrm{OH}\right)$, however catalase and glutathione can neutralise these species respectively. ${ }^{18}$

Type II reactions lead to the formation of singlet oxygen $\left({ }^{1} \mathrm{O}_{2}\right)$, a highly cytotoxic ROS that non-selectively oxidises biomolecules such as nucleic acids, proteins and lipids in order to inactivate the pathogen. ${ }^{17}$ Singlet oxygen formation occurs through the interaction of the excited triplet state PS with ground state triplet oxygen $\left({ }^{3} \mathrm{O}_{2}\right.$, Figure 1). The efficiency of the light absorption that leads to this event can be quantified through the measurement of the singlet oxygen quantum yield $\left(\Phi_{\Delta}\right)$. Effective defence mechanisms against singlet oxygen have not evolved within non-photosynthetic microorganisms, hence it is considered key to PDI of bacterial and viral pathogens. ${ }^{12,13}$

Due to its excellent photosensitising properties, abundance and lack of toxicity, riboflavin has several applications within blood product sterilisation in combination with ultraviolet (UV) light for effective PDI of pathogens prior to transfusion. ${ }^{18,19}$ More recently, it was shown to inactivate coronaviruses such as MERS and SARS-CoV-2. ${ }^{20-22}$ Similarly, it has been reported that riboflavin and FMN can inactivate bacteria, ${ }^{23}$ cancer cells $^{24}$ and parasites ${ }^{25}$ using either UV or blue $(440-460 \mathrm{~nm})$ light. Despite such promising studies, the design of flavin derivatives to understand structure-activity relationships towards PDI applications has not been explored, unlike prominent photosensitisers such as porphyrins. ${ }^{26}$ Previously, it was shown that functionalisation of flavins with amino groups, which introduce positive charge, can enhance PDI against both Gram-negative and Gram-positive bacteria as well as endospores. ${ }^{27,28}$ In general, cationic groups are known to improve the efficacy of PDI by coordination of the molecule to negatively charged phospholipid membranes. However, charge is not the only factor that contributes to the efficiency of PDI. For example, lipophilic flavin derivatives bearing multiple acetyl ester moieties have been shown to improve the efficacy against the Leishmania major parasite over analogous cationic ones, highlighting the importance of the cell membrane permeability. ${ }^{25}$

By considering the mechanism of PDI, as well as the biochemical structure of pathogens, we report the rational design of flavin derivatives (F1-4, Figure 2a) for pathogen inactivation. The prepared flavins demonstrate high efficacy and rapid inactivation of two model pathogens, a Gram-negative bacterium, E. coli BL21(DE3), and, murine hepatitis virus A59 strain (MHV-A59) ${ }^{29}$ often used as a surrogate for study of other coronaviruses such as SARS-Cov-2 despite the differences in transmission path, under white light irradiation (400-700 nm). In addition, the toxicity of the derivatives towards human fibroblast cells (WI-38) is explored, revealing that clear structure-activity relationships can be observed relating to the functionality of derivatives.

\section{Results}

\section{Synthesis and characterisation of flavins}

Flavins F1-4 (2A) were prepared according to Schemes S1 and S2 (see ESI). Inspired by the classical amphiflavins developed by Trissel, Schmidt and Hemmerich, ${ }^{30-32}$ we chose to include an alkyl chain $\left(C_{8}\right)$ within the structure to improve phospholipid membrane incorporation. Additionally, the flavin chromophore itself was substituted with bromo groups in the case of F3-4 in order to harness the heavy-atom effect that increases the rate of ISC from singlet to triplet excited states, thereby potentially enhancing photosensitised generation of singlet oxygen. ${ }^{33}$ Heavy atom substitution has been shown to improve both the rate of ISC and the singlet 
oxygen quantum yield $\left(\Phi_{\Delta}\right)$ in flavin derivatives used for synthetic photooxidation reactions. ${ }^{34}$ Although the heavy-atom effect has been used to boost photodynamic efficacy for other PS dyes in PDI applications, it has not yet been explored for flavin derivatives. ${ }^{35}$

To afford both methylated (F1-2) and brominated derivatives (F3-4), a Boc-protected ethylene amino component was first installed to the methylated or brominated arene core prior to cyclisation of the isoalloxazine ring system. Following $\mathrm{N}^{3}$-alkylation with an octyl chain on the isoalloxazine, N-Boc protecting groups were removed to yield the amino-functionalised F1 or F3 that were subsequently converted to guanidino moieties to achieve F2 or F4 respectively (Figure 2a). Guanidino groups were chosen as they are known to increase membrane coordination and penetration through strong guanidinium-phosphate $\mathrm{H}$-bonding and we hypothesised would enhance PDI efficacy.

The UV-Vis absorption spectra of the flavins in DMSO revealed very similar absorption properties at the $\lambda_{1}\left(S_{0} \rightarrow S_{1}\right)$ band but a blue shift of 10-13 nm for the higher energy $\lambda_{2}\left(S_{0} \rightarrow S_{2}\right)$ bands of the brominated compounds (F3 and F4) presumably due to the electron withdrawing effect of Br atoms (Figure 2b, Table 1). In terms of emission properties, the heavy-atom effect of bromination can be clearly observed with F3 and F4 exhibiting severely reduced emission intensity $\left(\Phi_{\mathrm{F}}<2 \%\right.$ in DMSO, Table 1$)$ when compared to the methylated F1 and $\mathbf{F} 2\left(\Phi_{\mathrm{F}}=13 \%\right.$ and $14 \%$ respectively in DMSO, Table 1$)$. This effect can also be observed when comparing the efficiency of ${ }^{1} \mathrm{O}_{2}$ production upon excitation, where brominated $\mathbf{F} \mathbf{3}$ and $\mathbf{F} \mathbf{4}$ demonstrate up to a $30 \%$ increase in activity over the methylated F1 and F2 in MeCN (Table 1), indicating their potential to be potent photodynamic agents. Despite this, F1 and F2 are still efficient ${ }^{1} \mathrm{O}_{2}$ photosensitisers comparable with riboflavin $\left(\Phi_{\Delta}=0.54 \pm\right.$ 0.07). However, a smaller $\Phi_{\Delta}$ value for guanidinylated F4 (62\%) is observed compared to aminated F3 (85\%) which could be explained by fast reverse ISC and/or solvent-dependent aggregation.

Table 1. Photophysical properties of flavins F1-4. $\lambda_{n}=S_{0} \rightarrow S_{n}$ absorption band, $\lambda_{\text {em }}=$ emission wavelength, $\varphi_{F}=$ fluorescence quantum yield, $\varphi_{\Delta}=$ singlet oxygen quantum yield.

\begin{tabular}{|c|c|c|c|c|c|}
\hline Flavin & $\lambda_{1}(\mathrm{~nm})^{\mathrm{a}}$ & $\lambda_{2}(\mathrm{~nm})^{\mathrm{a}}$ & $\lambda_{\mathrm{em}}(\mathrm{nm})^{\mathrm{a}}$ & $\varphi_{\mathrm{F}}^{\mathrm{b}}$ & $\varphi_{\Delta}^{c}$ \\
\hline F1 & 444 & 349 & 507 & $0.129 \pm 0.012$ & $0.49 \pm 0.12$ \\
\hline F2 & 446 & 350 & 512 & $0.141 \pm 0.010$ & $0.55 \pm 0.10$ \\
\hline F3 & 443 & 336 & 508 & $0.009 \pm 0.013$ & $0.85 \pm 0.09$ \\
\hline F4 & 444 & 340 & 513 & $0.011 \pm 0.010$ & $0.62 \pm 0.10$ \\
\hline
\end{tabular}

After successful synthesis and initial characterisation, we monitored the photostability of the compounds in PBS (1x, $\mathrm{pH} 7.4)$ which was used for PDI assays as distilled or ultrapure water destabilises bacterial cells and coronaviruses through osmotic pressures, ${ }^{36,37}$ thereby augmenting inactivation results. Flavins F1-4 and riboflavin (100 $\mu \mathrm{M}$ in PBS) were therefore irradiated with a 6200K white LED light source (18W, 400-700 nm, see 
ESI Figure S1 for emission spectrum) at an illuminance of $1 \times 10^{5} \mathrm{Ix}\left(35 \mathrm{~mW} / \mathrm{cm}^{2}\right.$ irradiance) to resemble typical daylight, ${ }^{38}$ and the changes in their UV-Vis absorption were monitored over time (see ESI, Figure S4). For riboflavin, rapid photodecomposition (80\%) was observed over 30 min irradiation which is known to be due to intramolecular dealkylation of the ribityl chain (Figure S4a, S4b). This yields lumichrome as the major degradation product which can only act as a PS under UV-irradiation. ${ }^{39}$ Amino-containing F1 and F3 exhibited around $40 \%$ and $60 \%$ degradation respectively after the same irradiation time (Figure S4a, S4c and S4e), whereas F2 and F4 degraded by approximately $10 \%$ and 30\% respectively (Figure S4a, S4d and S4f). These findings corroborate previous work showing amino-containing flavins photodegradation in the presence of phosphate ions which resulted in diminished bacterial PDI efficiency, ${ }^{40}$ however it appears that guanidino substitution improves photostability in this case. Interestingly, bromo-substituted flavins show higher rates of photodegradation which could be explained by their higher $\Phi_{\Delta}$ values resulting in greater ${ }^{1} \mathrm{O}_{2}$-induced degradation.

\section{Photodynamic inactivation of $E$. coli}

Having observed clear trends in the photophysical properties of methylated and brominated derivatives, we were interested to see how this would affect the PDI efficacy of F1-4 against pathogens. First, we investigated the inactivation of the Gram-negative bacterium, E. coli BL21(DE3). The cell envelope of Gram-negative bacteria presents a formidable barrier to antimicrobial compounds that consequently inhibits PDI efficacy compared to the analogous structure in Gram-positive bacteria. ${ }^{41}$ Following an initial 20 min incubation of the flavin compounds with E. coli in PBS at various concentrations in the dark, the mixture was irradiated $\left(1 \times 10^{5} \mathrm{Ix}\right)$ and the number of surviving colony forming units (CFUs) were determined. After $15 \mathrm{~min}\left(31.5 \mathrm{~J} / \mathrm{cm}^{2}\right.$ light dose) of irradiation, no inactivation was observed at $1 \mu \mathrm{M}$ for either riboflavin or F1-4. At $10 \mu \mathrm{M}$ approximately $1 \log _{10}$ reduction of $E$. coli CFUs was observed for $\mathbf{F} 2$ and a $2.8 \log _{10}$ reduction in the presence of F4 (Figure 3a, Table S1). At higher concentrations, this effect was greatly enhanced with both F2 and F4 exhibiting >6.0 $\log _{10}$ reduction of $\mathrm{CFU} / \mathrm{mL}$ at $100 \mu \mathrm{M}$. From these data, it can be derived that $11 \mu \mathrm{M}$ of F4 and $42 \mu \mathrm{M}$ of F2 can achieve $>3$ log reduction ( $>99.9 \%$ ) of bacterial load which is considered a minimum level of decontamination according to EMA guidance, whereas $>6$ logs is the highest. ${ }^{42}$

Interestingly, riboflavin and F1 demonstrated no activity at $100 \mu \mathrm{M}$, whereas F3 exhibited a $1.8 \log _{10}$ reduction in bacterial load. To ensure effective PDI was occurring, the flavin compounds were incubated in the dark under the same experimental irradiation conditions (100 $\mu \mathrm{M}, 15 \mathrm{~min}$ ) to reveal no bacterial toxicity (see ESI, Figure S5). The rate of $E$. coli inactivation over time was then monitored at a $100 \mu \mathrm{M}$ flavin concentration revealing an extremely rapid reduction of CFUs in the presence of F4, facilitating $>6.0 \log _{10}$ reduction after just 5 min of irradiation (10.5 J/cm² light dose). Similarly, F2 shows effective bactericidal activity with a $3.4 \log _{10}$ reduction $(>99.9 \%)$ after 5 min irradiation. A closer investigation into the speed of F4's activity at $100 \mu \mathrm{M}$ showed that after only $1 \mathrm{~min}$ of irradiation (2.10 J/cm² light dose) a $4.1 \log _{10}$ reduction (>99.99\%) of bacteria was achieved (Figure 3b).

These data clearly show that the introduction of a guanidino moiety, as in the case of F2 and F4, greatly increases the flavin's PDI efficacy against the bacterium when compared to amino-containing F1 and F3. This may be attributed to better photostability in PBS, as well as the guanidino group facilitating better coordination 
to the cytoplasmic phospholipid membrane resulting in improved permeability and lipid peroxidation under irradiation. ${ }^{43}$ In addition, the replacement of methyl substituents with bromines improves efficacy when the same cationic group is compared. This can be explained by more efficient generation of singlet oxygen, as predicted by their $\Phi_{\Delta}$ values shown in Table 1. As a result, greater lipid and biomolecule oxidation can be achieved to inactivate the pathogen. It should also be noted that the inclusion of bromine atoms increases the lipophilicity of the molecule which can further improve cell membrane permeability and incorporation. However, despite having the highest predicted $\Phi_{\Delta}$ value (85\%), F3 did not outperform F2 $\left(\Phi_{\Delta}=55 \%\right)$ which demonstrates the overarching impact of guanidino substitution.

To gain initial mechanistic insight, we investigated the cellular localisation of fluorescent riboflavin, F1 and F2 by structured illumination microscopy (SIM) (Figure 3c, see ESI Figures S6-8). After incubation with the flavin compounds $(100 \mu \mathrm{M})$, large field of view images revealed marked differences in the number and degree of fluorescent bacterial populations (see ESI Figures S6-8). Although the exact quantification of flavin uptake was not possible due to uncharacterised optical properties in a complex biological environment, the qualitative comparison of F2 and F1 (which have similar $\Phi_{F}$ in DMSO), revealed a higher number of fluorescent bacteria with bright fluorescent intensity after incubation with F2, especially within membranes (Figure 3c, Figure S6 and S7). This indicates that guanidino substitution does improve uptake, however the distribution of fluorescent intensity was not homogeneous across different bacterial cells which could be related to the amphiphilicity of F2, resulting in variable uptake due to aggregation (Figure S6). For hydrophilic riboflavin, very weak fluorescent populations were observed, most likely explained by the controlled transport of the compound through outer membrane porins of the bacterium and therefore unable to bind effectively to the outer or cytoplasmic membranes (Figure 3c, Figure S8). This therefore helps to explain the lack of PDI efficacy observed when using riboflavin.

Furthermore, we monitored the pDNA (pUC18) cleavage in the presence of the flavins $(10 \mu \mathrm{M})$ under irradiation which indicated guanidino derivatives possess superior photocleavage ability (Figure $3 d$ ). The photocleavage of supercoiled (SC) to nicked coiled (NC) pDNA structures was far enhanced for F2 and F4 (39\% and 21\% respectively) when compared to F1 and F3 ( $\leq 3 \%$ ) after irradiation for 15 min (Figure $\mathbf{3 d}$ ). This difference in activity could be explained by favourable guanidinium-phosphate interactions that increase the likelihood of electron transfer events between flavin and guanosine which are known to primarily contribute to DNA cleavage alongside ${ }^{1} \mathrm{O}_{2}$-mediated oxidation. ${ }^{44,45}$ Therefore, even if the amino-flavin compounds diffuse into the cytoplasm, it is unlikely that damage to constituent nucleic acids would contribute to PDI of the pathogen. Our control compound, riboflavin (Rbf) also exhibits photocleavage of the plasmid (11\%) which has been reported previously, ${ }^{44,45}$ however it is clear from the SIM data that, due to its hydrophilic nature and lack of cationic substituent, little cell uptake of the compound is achieved for this to contribute to PDI.

Collectively, this initial mechanistic study demonstrates that even in the case of increased singlet oxygen production, the key component to achieve effective Gram-negative bactericidal activity under irradiation is the presence of the guanidino group, which facilitates enhanced cell uptake and nucleic acid degradation.

\section{Photodynamic inactivation of murine hepatitis virus (MHV-A59)}

Encouraged by the identification of such highly effective guanidino-flavins for bacterial inactivation, we were interested to see if the trend would be similar for coronaviruses, specifically, murine hepatitis virus A59 strain 
(MHV-A59) which is often used as a surrogate for other coronaviruses. ${ }^{4}$ It has already been demonstrated that riboflavin can effectively inactivate both enveloped and non-enveloped viruses in blood products using UV light, ${ }^{20-23}$ but much lower efficacy was observed using visible light $\left(0.4 \times 10^{5} \mathrm{Ix}, 0.5-2 \mathrm{~h}\right)$ against hepatitis $B$ virus (HBV). ${ }^{46,47}$ In order to evaluate possible applicability towards virus-inactivating surface coatings or textiles, we used an in vitro TCID $_{50}$ assay to evaluate the viral titre of MHV-A59 through inoculation into murine fibroblast $17 \mathrm{Cl}-1$ cells after irradiation at varying concentrations of flavin in PBS. The cytotoxicity of the flavins towards this cell line was first investigated by $24 \mathrm{~h}$ incubation MTS assay allowing us to obtain a working concentration range of $\leq 10 \mu \mathrm{M}$ for the in vitro $\operatorname{TCID}_{50}$ assay to evaluate viral PDI efficacy (see ESI Figures S9 and Table S3 for $17 \mathrm{Cl}-1$ cytotoxicity data).

We started our investigation by varying the concentration of flavin (1-10 $\mu \mathrm{M}$ in PBS) with 10 min of white LED exposure $\left(21.0 \mathrm{~J} / \mathrm{cm}^{2}\right.$ light dose, Figure $\left.4 \mathrm{a}\right)$. Even at $1 \mu \mathrm{M}$, the brominated guanidino flavin $\mathbf{F 4}$ demonstrated a $3.8 \log _{10}$ reduction in viral titre, while F2 and F3 showed $2.1 \log _{10}$ reduction (Figure $4 \mathrm{a}$, Table S3). It should be noted that reductions of the order of 4 logs or more (>99.99\%) are considered highly effective by EMA guidance and that a $>1 \log _{10}$ reduction in necessary to be considered reliable. ${ }^{48}$ At the same concentration, riboflavin achieved a $1.2 \log _{10}$ reduction in titre, however F1 showed no effect under these conditions. Nevertheless, the activity of all flavins improved by the increase of their concentrations resulting in viral load reductions of $>99.9 \%$ in the presence of riboflavin or $\mathbf{F 2}$, and $>99.99 \%$ with $\mathbf{F 3}$ or F4 at $5 \mu \mathrm{M}$. Further increase in concentration (to 10 $\mu \mathrm{M})$ only substantially improved virucidal activity for F4 $\left(>5 \log _{10}\right)$. The irradiation time was then explored for 10 $\mu \mathrm{M}$ flavin to reveal high degrees of inactivation $\left(\geq 5 \log _{10}\right)$ for F2, F3 and F4 when irradiated for 15 min (31.5 $\mathrm{J} / \mathrm{cm}^{2}$ light dose) whereas shorter irradiation times (5 min, $10.5 \mathrm{~J} / \mathrm{cm}^{2}$ light dose) still provided efficient inactivation of MHV-A59 $\geq 3 \log _{10}$ steps using those same flavins (Figure $4 \mathbf{b}$ ). It should be noted that variability within all experiments was noticeably high which is typical for $\operatorname{TCID}_{50}$ assays. ${ }^{49}$ However, no reliable log reduction of MHV-A59 was observed when irradiated without flavin in PBS containing 0.1\% DMSO (Figure 4b). To confirm that the mechanism of viral inactivation by the flavins was dependent on light, dark control experiments $\left(10 \mu \mathrm{M}, 10\right.$ min incubation) resulted in no effective virucidal activity being observed $\left(>1 \log _{10}\right.$ reduction, Figure $\mathbf{4 c}$ ).

The activity of the flavin derivatives towards viral inactivation show a different trend than previously observed with our model bacterium. For example, in the presence of riboflavin under the same conditions, effective PDI of MHV-A59 was measured whereas there was no activity towards $E$. coli. Therefore, it can be assumed that the requirements for a PS to interact and/or diffuse through the coronavirus membrane are less dependent on lipophilicity or the presence of cationic charge. Despite this, our flavin derivatives again show that guanidino group incorporation improves antiviral PDI activity when compared to amino groups. However, bromination now seems to play a more important role in viral deactivation as brominated amino F3 outperformed methylated guanidino F2.

To try and explain our findings, we investigated the interaction of the flavins with RNA to elucidate how its lightinduced cleavage could lead to coronavirus inactivation, a model ssRNA ( $1 \mathrm{k} \mathrm{nt})$ was irradiated $\left(1 \times 10^{5} \mathrm{Ix}\right)$ in the presence of $10 \mu \mathrm{M}$ flavin in PBS for 15 min and the extent of cleavage was observed via agarose gel electrophoresis (Figure 4d). It was observed that F1 exhibits the least RNA photocleavage (12\%) whereas riboflavin shows greater activity $(28 \%)$ which corroborates the result of viral inactivation and confirms previous 
findings.${ }^{50-52}$ Similar to our results with pDNA, guanidino-containing F2 and F4 show the best photocleavage ability ( $46 \%$ and $42 \%$ respectively). Interestingly, F3 also exhibits effective cleavage of RNA (35\%) which was not observed in the case of pDNA (Figure 3d). This can be rationalised by the mechanism of flavin-mediated RNA photocleavage which has been previously shown to depend more upon ${ }^{1} \mathrm{O}_{2}$ oxidation than electron transfer events between flavin and nucleobase. ${ }^{50-52}$ Taken together, it can be concluded that efficient ${ }^{1} \mathrm{O}_{2}$ production is key to viral PDI using flavin derivatives.

\section{In vitro toxicity towards human cells}

Finally, before considering the applications of our flavin derivatives for PDI of topical pathogen infections, surface coatings or textiles, we investigated their impact on human cells. In general, an ideal photosensitiser for these applications should show no cytotoxic effects on human cells in the dark and limited effects given the same irradiation conditions used for inactivation of the target pathogen, also referred to as a therapeutic window. ${ }^{12}$ Accordingly, the inherent cytotoxicity of the flavins was evaluated through incubation with human lung fibroblast cells (WI-38). A $24 \mathrm{~h}$ MTS assay was used to calculate inhibitory concentration values $\left(\mathrm{IC}_{50}\right)$ of $>100 \mu \mathrm{M}$ for riboflavin and guanidino-functionalised $\mathbf{F} 2$ and $\mathbf{F} 4$ which can therefore be considered as non-toxic (Figure 5a, Table S4). On the other hand, amino-functionalised F1 and F3 had IC $\mathrm{C}_{50}$ values of $96.1 \mu \mathrm{M}$ and 30.9 $\mu \mathrm{M}$ respectively (Table S4). It has been shown previously that the cytotoxicity of amino-containing compounds may be derived from an increase in intracellular amine oxidase activity that induces excess oxidative stress leading to cell apoptosis. ${ }^{53}$

We then studied the light-induced toxicity of the flavins towards the WI-38 fibroblast cells by observing decreases in cell viability over irradiation time using an MTS assay (Figure 5b). Interestingly, the cytotoxicity trends observed in the dark after $24 \mathrm{~h}$ are reversed when exposed to light $\left(1 \times 10^{5} \mathrm{Ix}\right)$ over shorter time periods. For example, after 15 min of irradiation, $10 \mu \mathrm{M}$ of methylated guanidino $\mathbf{F} 2$ induces $\sim 75 \%$ reduction in cell viability. Under the same conditions, brominated guanidino F4 exhibits less of an effect with $\sim 20 \%$ reduction in cell viability. The degree of variability in these experiments could be attributed to the amphiphilic nature of the flavins leading to aggregation of the compounds in aqueous conditions. On the other hand, riboflavin, aminofunctionalised $\mathbf{F} \mathbf{1}$ and $\mathbf{F} \mathbf{3}$ display no decrease in cell viability over $\mathbf{1 5}$ min irradiation. These data show that despite being non-toxic to the cells in the dark, the guanidino-functionalised flavins can induce cytotoxicity upon irradiation most likely through similar mechanisms discussed for pathogens. It is our ongoing work to understand the differences in light induced cytotoxicity that were observed between methylated and brominated guanidino flavin derivatives. Nevertheless, F4 could be suitable for further PDI applications thanks to effective bacterial and viral inactivation (>3 $\log _{10}$ reduction after 15 min irradiation at $11 \mu \mathrm{M}$ and $1 \mu \mathrm{M}$ respectively) coupled with low toxicity to human fibroblast cells under the same conditions or in the dark.

\section{Conclusion}

In summary, we have rationally designed a set of flavin derivatives containing functional groups that significantly improve visible light photodynamic inactivation of pathogens. By incorporating a guanidino moiety into the flavin structure (F2 and F4), the inactivation efficacy against Gram-negative bacteria, E. coli BL21(DE3) in PBS, was remarkably enhanced compared to natural riboflavin and amino variants (F1 and F3). This was justified by enhanced cell uptake and pDNA cleavage facilitated by this group. Bromination of the structure (F3 
and F4) improved singlet oxygen production via the heavy-atom effect which generally led to improved PDI of $E$. coli, however the incorporation of a guanidino substituent dominated the outcome. Very effective inactivation was achieved with F4, whereby $1 \mathrm{~min}$ of visible irradiation $\left(2.10 \mathrm{~J} / \mathrm{cm}^{2}\right.$ light dose) reduced the bacterial load by $>4 \log _{10}$ steps.

It was found that less selectivity was placed upon the presence of a guanidino moiety when evaluating the PDI against the SARS-CoV-2 surrogate, MHV-A59 in PBS. This was evidenced by brominated amino F3 having a higher efficacy compared to methylated guanidino F2 which was attributed to greater RNA cleavage via ${ }^{1} \mathrm{O}_{2}$ oxidation. The highest viral PDI was achieved by F4 where $>4 \log _{10}$ reduction in titre was achieved after 10 min of visible irradiation $\left(21.0 \mathrm{~J} / \mathrm{cm}^{2}\right.$ light dose) in the presence of $5 \mu \mathrm{M}$ compound. Toxicity studies using the novel flavins towards human fibroblast cells (WI-38) further confirmed that F4 could be a suitable candidate for PDI applications against both bacteria and viruses.

Overall, we hope this study inspires further exploration of riboflavin's structure to design new generations of photosensitiser compounds to treat topical bacterial infections, enabling the design of antiviral surface coatings, sprayable hybrid materials to provide the surface protection, as well as fabrics capable of efficient visible light pathogen inactivation. Such fabrics could be used to manufacture protecting suits for medical staff, as well as curtains and bedding used in intensive care units where the sterile conditions are of the outmost importance.

\section{Methods}

\section{Synthesis and characterisation of flavins}

Detailed experimental methods of synthesis and characterisation of flavins can be found in the Supplementary Information.

\section{Photodynamic inactivation of $E$. coli}

An adapted method reported by Maisch et al. was used to evaluate the phototoxicity of flavin compounds towards E. coli BL21(DE3). ${ }^{27}$

After an overnight aerobic culture in LB broth at $37^{\circ} \mathrm{C}$ with shaking at $225 \mathrm{rpm}$, the resulting E. coli were centrifuged at 2,500 rpm for 10 minutes. The pellet was resuspended in PBS (Sigma, 1x, pH 7.4) to an $\mathrm{OD}_{600}$ value of $0.6\left(\sim 1 \times 10^{8}\right.$ bacteria per $\left.\mathrm{ml}\right)$. Bacterial solutions were then combined with varying concentrations of F14 and riboflavin $(0,1,10,100 \mu \mathrm{M})$ dissolved in PBS in a 1:1 ratio and left to incubate for 20 minutes in dark. Aliquots of negative controls (0.5\% DMSO in PBS; PBS only) and $100 \mu \mathrm{M}$ flavin-bacteria samples were covered in foil to remain in dark, while all samples were plated onto a 96-well plate and exposed to white LED irradiation $\left(1 \times 10^{5} \mathrm{Ix}\right)$ for 15 minutes. After 15 minutes of irradiation or darkness, each sample was serially diluted to its respective, appropriate dilution and plated onto an LB agar plate with $50 \mu \mathrm{g} / \mathrm{mL}$ kanamycin. Plates were incubated overnight at $37^{\circ} \mathrm{C}$ in the dark. Survival of bacteria was determined by counting colony forming units (CFUs) the next day.

For time point data, aliquots of $100 \mu \mathrm{M}$ flavin-bacteria solutions were taken at 5-minute increments of irradiation

$(0,5,10$, and 15 minutes) and evaluated similarly as above. Time point experiment with one-minute increments, 
between 0 and 5 minutes, were conducted for $100 \mu \mathrm{M}$ F4-bacteria samples.

All data was generated with technical replicates and biological triplicates.

All statistical analysis was done with Graphpad Prism 9. One-way ANOVA with Tukey's multiple comparisons test, was performed to evaluate the impact of flavin concentration compared to the control (PBS). Two-way ANOVA with Bonferroni's multiple comparison test, was performed to evaluate the impact of light given the same flavin concentration with different irradiation conditions (dark and light). Significance levels are defined as the following: $n s$ for $p>0.05$, * for $p \leq 0.05$, ** for $p \leq 0.01$, *** for $p<0.001$, and $* \star \star \star$ for $p<0.0001$.

\section{Photodynamic inactivation of murine hepatitis virus (MHV-A59)}

\section{Cell Culture}

17Cl-1 murine fibroblasts cells and murine hepatitis virus A59 strain (MHV-A59) were kindly gifted by Professor lan Goodfellow's lab (Department of Pathology, University of Cambridge, UK). $17 \mathrm{Cl}-1$ cells were resurrected from cold-storage cell banks and propagated using routine cell culture protocols in complete growth medium (Dulbecco's modified Eagle's medium low glucose $1 \mathrm{~g} / \mathrm{L}$ (DMEM, Life Technologies) supplemented with 5\% foetal bovine serum (Merck), 6\% tryptose phosphate broth (Merck), 1x non-essential amino acids (Gibco), 1x antibioticantimycotic (Thermo Fisher Scientific) and 1x L-Glutamine (Gibco). 17Cl-1 cells were seeded (100 $\mu$ l per well) into 96-well plates (untreated, flat bottom) at $1 \times 10^{4}$ cells/well at $37^{\circ} \mathrm{C}$ for 24 hours in $5 \% \mathrm{CO}_{2}$ prior to the day of testing.

\section{Virus Propagation}

MHV-A59 was propagated in 17Cl-1 cells at a multiplicity of infection (MOI) of 0.01 TCID $_{50}$ per cell. MHV-A59 was clarified by centrifugation at $3000 \mathrm{rpm}$ for $10 \mathrm{~min}$ and stored in aliquots at $-80^{\circ} \mathrm{C}$. The $\operatorname{TCID}_{50}$ was calculated using the Reed and Muench method. ${ }^{54}$

\section{Flavin Treatment of MHV-A59}

The efficacy of the flavins were enumerated utilizing modified methods from ISO 18184 and Leibowitz et al. ${ }^{55}$ Flavins F1-4 and riboflavin, were solubilized in DMSO. $100 \mu \mathrm{M}$ working stock solutions were then created by diluting each DMSO solution into PBS. To control for the effect of DMSO on MHV-A59, DMSO was diluted into PBS at the same concentration. A white light apparatus was set up to cover a 4 x 5 (row x column) area of a 96well plate with the same level of light intensity $\left(1 \times 10^{5} \mathrm{Ix}\right)$. The stock solutions were then diluted in replicates of four to a total of $180 \mu \mathrm{l}$ in PBS at 1,5 , and $10 \mu \mathrm{M}$ concentrations for each sample. Virus stocks of MHV-A59 were thawed on ice prior to use. $20 \mu \mathrm{l}$ of MHV-A59 stock (1.4 x $109 \mathrm{PFU} / \mathrm{ml})$ was then mixed into each well, and the 4 $x 5$ well matrix was treated with the light or incubated in the dark at room temperature for a specified time $(5,10$, or 15 minutes).

\section{Virus Recovery and $T_{C I} D_{50}$ Assay MHV-A59}

After treatment, the samples were mixed into $320 \mu$ of Gibco Difco ${ }^{\text {TM }}$ Beef Extract $(1.5 \%$ w/v beef extract in $\mathrm{ddH}_{2} \mathrm{O}$; Life Technologies) and rolled for 15 minutes to chelate any free ions in the solution. The samples then 
were serially diluted $\left(10^{0}-10^{-7}\right.$ ) in infectivity media (Dulbecco's modified Eagle's medium low glucose $1 \mathrm{~g} / \mathrm{L}$ (DMEM, Life Technologies) supplemented with 2.5\% fetal bovine serum (Merck), 3\% tryptose phosphate broth (Merck), 1x non-essential amino acids (Gibco), 1x antibiotic-antimycotic (Thermo Fisher Scientific) and 1x LGlutamine (Gibco]) and added in triplicate to $17 \mathrm{Cl}-1$ cell plates. For each sample or control there were 4 replicate treatments (in light or dark); each treatment replicate was plated in triplicate. Infected 17Cl-1 plates were incubated at $37^{\circ} \mathrm{C}$ in a $5 \% \mathrm{CO}_{2}$ atmosphere for 24 hours. Plates were scored for cytopathic effect (CPE) by microscopy and viral titres were determined by the Reed and Muench $50 \%$ tissue culture infectious dose $\left(\operatorname{TCID}_{50}\right)$ end point method. ${ }^{54}$ Outliers were removed from the four replicates using an outlier test. The full protocol was repeated in triplicate on different days to account for any variability in the assay.

\section{Nucleic acid photocleavage}

General procedure: Riboflavin (Rbf) or flavin F1-4 (10 $\mu \mathrm{M}$ from DMSO stock solutions) were mixed with either pUC18 DNA (Thermo Scientific, $0.50 \mu \mathrm{g})$ or EGFP-encoding mRNA ( 1k nt) as the ssRNA model (TriLink Biotech, $0.88 \mu \mathrm{g})$ dissolved in PBS $(40 \mu \mathrm{L})$ to give a final DMSO concentration of $0.1 \%$. A $20 \mu \mathrm{L}$ aliquot was taken after 15 min of white LED irradiation $\left(1 \times 10^{5} \mathrm{Ix}\right)$. Prior to loading, $2 \mu \mathrm{L}$ of $6 \mathrm{X}$ Orange-G loading dye in glycerol were added to the aliquot and mixed vigorously. The gel was electrophoresed at $80 \mathrm{~V}$ for $35 \mathrm{~min}$ with $1 \%$ agarose gels in $1 \mathrm{X}$ Tris-acetate-EDTA (TAE) buffer. Gels were then imaged in a Syngene G:BOX Gel Documentation System and band \% quantification was performed using ImageJ Gel Analyzer.

\section{In vitro toxicity towards human cells}

\section{Cell Culture}

Normal human lung fibroblasts, WI-38, were purchased from American Type Culture Collection (ATCC) and murine fibroblast cell line, 17Cl-1, was kindly provided by lan Goodfellow's lab (Department of Pathology, University of Cambridge, UK). WI-38 cells were cultured in Dubelco's modified essential medium (Gibco) with 10\% fetal bovine serum (FBS, Gibco) containing penicillin $(50 \mathrm{IU} / \mathrm{mL})$ and streptomycin $(50 \mu \mathrm{g} / \mathrm{mL})$ (Thermo Fisher Scientific). $17 \mathrm{Cl}-1$ cells were cultured as described above. All Cell lines were maintained in a humidified environment at $37^{\circ} \mathrm{C}$ with $5 \% \mathrm{CO}_{2}$ and routinely tested to confirm the absence of Mycoplasma. All in vitro experiments were conducted between $60 \%$ and $80 \%$ confluent cultures at passage 6-15 (WI-38) and 25-35 $(17 \mathrm{Cl}-1)$.

\section{MTS viability assay}

The effect on cell viability of WI-38 and 17Cl-1 cells after treatment with F1-F4 and riboflavin was determined using the commercially available MTS [3-(4,5-dimethylthiazol-2-yl)-5-(3-carboxymethoxyphenyl)-2-(4sulfophenyl)- $2 \mathrm{H}$ tetrazolium] assay (Promega). The MTS tetrazolium compound is reduced by cells into a coloured formazan product which is soluble in cell culture media. It can be detected colorimetrically between 450-540 nm with the measured absorbance directly proportional to the amount of metabolically active cells in culture. Cells were seeded into clear 96-well plates containing 10000 cells/well in $100 \mu \mathrm{L}$ complete growth medium and cultured for 24 hours at $37^{\circ} \mathrm{C}$ and $5 \% \mathrm{CO}_{2}$. Subsequently, cells were treated with varying concentrations of F1-F4 and riboflavin (0.01-100 $\mu \mathrm{M})$ dissolved in complete growth media containing $0.1 \%$ DMSO. After further 24 hours incubation at $37^{\circ} \mathrm{C}$ and $5 \% \mathrm{CO}_{2}, 20 \mu \mathrm{L}$ of CelTiter $96^{\circledR} \mathrm{AQ}_{\text {ueous }}$ One Solution 
(Promega) was added into each well and incubated at $37^{\circ} \mathrm{C}, 5 \% \mathrm{CO}_{2}$ for $1-4$ hours, according to the manufacturer's instruction. The absorbance of each well was measured at $490 \mathrm{~nm}$ using a plate reader (Spark, Tecan). Control measurements included negative control of cells with DMEM, cells with DMEM containing $0.1 \%$ DMSO, cell-free culture media (blank) and cell-free sample dilutions in culture media to evaluate potential sample interferences with MTS assay. All experiments were conducted in biological triplicates. The percentage cell viability was calculated according to equation (1):

$$
\text { Cell viability }(\%)=100 \times\left(\frac{\text { Absorbance of treated cells-Absorbance of blank }}{\text { Absorbance of control-Absorbance of blank }}\right)(1)
$$

An adapted method reported by Maisch et al. was used to conduct phototoxicity studies on human lung fibroblasts, WI-38. ${ }^{27}$ Cells were seeded into 96-well plates containing 10000 cells/well in $100 \mu \mathrm{L}$ complete growth medium and cultured for 24 hours at $37^{\circ} \mathrm{C}$ and $5 \% \mathrm{CO}_{2}$. On the next day, the growth media was removed, and cells were treated with $10 \mu \mathrm{M}$ F1-F4 and riboflavin dissolved in DMEM without serum and phenol red (Gibco) containing $0.1 \%$ DMSO. The resulting mixtures were then incubated in the dark for 5 min and then either illuminated with $1 \times 10^{5}$ Ixfor 5, 10 or $15 \mathrm{~min}$, or incubated further in the dark for 15 min (dark control). After irradiation, the flavin solutions were removed and $100 \mu \mathrm{L}$ of DMEM with $10 \%$ FBS and without phenol red was added to each well and incubated over night at $37^{\circ} \mathrm{C}$ and $5 \% \mathrm{CO}_{2}$. Subsequently, $20 \mu \mathrm{L}$ of MTS reagent was added into each well and incubated at $37^{\circ} \mathrm{C}$ and $5 \% \mathrm{CO}_{2}$ for $1-4$ hours and the absorbance of each well was measured at $490 \mathrm{~nm}$ using a plate reader. Control measurements included negative control untreated cells with light and in the dark containing 0.1\% DMSO and cells treated with flavins for 15 min in the dark. All experiments were conducted in biological triplicates. The percentage cell viability was calculated according to equation (1).

\section{Declarations}

\section{Data availability}

The data that support the findings of this study are available from the corresponding author upon request.

\section{Acknowledgements}

This work was funded by EPSRC grant EP/V029762/1. LC would like to acknowledge support of the Leverhulme Trust grant RPG-2019-266. IM and CFK wish to acknowledge EPSRC grants EP/L015889/1 and EP/H018301/1. COF also acknowledges EPSRC grant EP/L015889/1 and the support by AstraZeneca in the framework of Project Beacon. AB acknowledges support of EPSRC IRC project EP/S009000/1. We wish to thank Ms Eleanor Hall for technical assistance with cell culture as well as the staff of NMR and MS facilities in the Department of Chemistry, the University of Cambridge for their assistance.

\section{Author Contributions}

Conceptualisation: L.C. and L.F. Investigation, methodology, data curation, formal analysis and visualisation: L.C., J.H.L., S.M., G.C.M., S.S., A.B.P., C.O.F. and I.M. Supervision and funding acquisition: C.F.K., G.C. and L.F. Writing - original draft: L.C. Writing - review \& editing: J.H.L., S.M., G.C.M., S.S., A.B.P., C.O.F., I.M., C.F.K., G.C. and L.F. 


\section{Conflicts of interest}

The authors declare no competing interests.

\section{References}

1. Walsh, C. Flavin Coenzymes: At the Crossroads of Biological Redox Chemistry. Acc. Chem. Res. 13, 148-155 (1980).

2. Massey, V. The chemical and biological versatility of riboflavin. in Biochemical Society Transactions vol. 28 283-296 (2000).

3. Walsh, C. T. \& Wencewicz, T. A. Flavoenzymes: Versatile catalysts in biosynthetic pathways. Natural Product Reports vol. 30 175-200 (2013).

4. Baker Dockrey, S. A. \& Narayan, A. R. H. Flavin-dependent biocatalysts in synthesis. Tetrahedron 75, 11151121 (2019).

5. Kennis, J. T. M. \& Mathes, T. Molecular eyes: Proteins that transform light into biological information. Interface Focus vol. 3 (2013).

6. Cashmore, A. R. Cryptochromes: Enabling plants and animals to determine circadian time. Cel/vol. 114 537-543 (2003).

7. Masuda, S. Light detection and signal transduction in the BLUF photoreceptors. Plant and Cell Physiology vol. 54 171-179 (2013).

8. Losi, A. \& Gärtner, W. Solving Blue Light Riddles: New Lessons from Flavin-binding LOV Photoreceptors. Photochemistry and Photobiology vol. 93 141-158 (2017).

9. König, B., Kümmel, S., Svobodová, E. \& Cibulka, R. Flavin photocatalysis. Phys. Sci. Rev. 3, (2019).

10. Graml, A., Neveselý, T., Jan Kutta, R., Cibulka, R. \& König, B. Deazaflavin reductive photocatalysis involves excited semiquinone radicals. Nat. Commun. 11, 1-11 (2020).

11. Pokluda, A. et al. Robust Photocatalytic Method Using Ethylene-Bridged Flavinium Salts for the Aerobic Oxidation of Unactivated Benzylic Substrates. Adv. Synth. Catal. 363, 4371-4379 (2021).

12. Cieplik, F. et al. Antimicrobial photodynamic therapy-what we know and what we don't. Critical Reviews in Microbiology vol. 44 571-589 (2018).

13. Wiehe, A., O'brien, J. M. \& Senge, M. O. Trends and targets in antiviral phototherapy. Photochem. Photobiol. Sci. 18, 2565-2612 (2019).

14. Maisch, T. Resistance in antimicrobial photodynamic inactivation of bacteria. Photochem. Photobiol. Sci. 14, 1518-1526 (2015).

15. Wainwright, M. et al. Photoantimicrobials-are we afraid of the light? The Lancet Infectious Diseases vol. 17 e49-e55 (2017).

16. Sabino, C. P. et al. Light-based technologies for management of COVID-19 pandemic crisis. Journal of Photochemistry and Photobiology B: Biology vol. 212111999 (2020).

17. Baptista, M. S. et al. Type I and Type II Photosensitized Oxidation Reactions: Guidelines and Mechanistic Pathways. Photochemistry and Photobiology vol. 93 912-919 (2017). 
18. Goodrich, R. P., Edrich, R. A., Li, J. \& Seghatchian, J. The Mirasol ${ }^{T M}$ PRT system for pathogen reduction of platelets and plasma: An overview of current status and future trends. Transfus. Apher. Sci. 35, 5-17 (2006).

19. Marschner, S. \& Goodrich, R. Pathogen reduction technology treatment of platelets, plasma and whole blood using riboflavin and UV light. Transfus. Med. Hemotherapy 38, 8-18 (2011).

20. Keil, S. D., Bowen, R. \& Marschner, S. Inactivation of Middle East respiratory syndrome coronavirus (MERSCoV) in plasma products using a riboflavin-based and ultraviolet light-based photochemical treatment. Transfusion 56, 2948-2952 (2016).

21. Ragan, I., Hartson, L., Pidcoke, H., Bowen, R. \& Goodrich, R. Pathogen reduction of SARS-CoV-2 virus in plasma and whole blood using riboflavin and UV light. PLoS One 15, e0233947 (2020).

22. Keil, S. D. et al. Inactivation of severe acute respiratory syndrome coronavirus 2 in plasma and platelet products using a riboflavin and ultraviolet light-based photochemical treatment. Vox Sang. 115, 495-501 (2020).

23. Wong, T. W., Cheng, C. W., Hsieh, Z. J. \& Liang, J. Y. Effects of blue or violet light on the inactivation of Staphylococcus aureus by riboflavin-5'-phosphate photolysis. J. Photochem. Photobiol. B Biol. 173, 672680 (2017).

24. Edwards, A. M., Barredo, F., Silva, E., De loannes, A. E. \& Becker, M. I. Apoptosis induction in nonirradiated human HL-60 and murine NSO/2 tumor cells by photoproducts of indole-3-acetic acid and riboflavin. Photochem. Photobiol. 70, 645-649 (1999).

25. Silva, A. V. et al. Riboflavin derivatives for enhanced photodynamic activity against Leishmania parasites. Tetrahedron 71, 457-462 (2015).

26. Kou, J., Dou, D. \& Yang, L. Porphyrin photosensitizers in photodynamic therapy and its applications. Oncotarget 8, 81591-81603 (2017).

27. Maisch, T. et al. Fast and effective photodynamic inactivation of multiresistant bacteria by cationic riboflavin derivatives. PLoS One 9, (2014).

28. Eichner, A. et al. Fast and effective inactivation of Bacillus atrophaeus endospores using light-activated derivatives of vitamin B2. Photochem. Photobiol. Sci. 14, 387-396 (2015).

29. Pendyala, B., Patras, A., Pokharel, B. \& D’Souza, D. Genomic Modeling as an Approach to Identify Surrogates for Use in Experimental Validation of SARS-CoV-2 and HuNoV Inactivation by UV-C Treatment. Front. Microbiol. 11, 2406 (2020).

30. Michel, H. \& Hemmerich, P. Substitution of the flavin chromophore with lipophilic side chains: A novel membrane redox label. J. Membr. Biol. 60, 143-153 (1981).

31. Schmidt, W. Fluorescence Properties of Isotropically and Anisotropically Embedded Flavins. Photochem. Photobiol. 34, 7-16 (1981).

32. Trissl, H. W. Studies on the incorporation of fluorescent pigments into bilayer membranes. BBA - Biomembr. 367, 326-337 (1974).

33. Koziar, J. C. \& Cowan, D. O. Photochemical Heavy-Atom Effects. Acc. Chem. Res. 11, 334-341 (1978).

34. Korvinson, K. A. et al. Improved Flavin-Based Catalytic Photooxidation of Alcohols through Intersystem Crossing Rate Enhancement. J. Phys. Chem. A 120, 7294-7300 (2016).

35. Gorman, A. et al. In vitro demonstration of the heavy-atom effect for photodynamic therapy. J. Am. Chem. Soc. $126,10619-10631$ (2004). 
36. Wood, J. M. Bacterial responses to osmotic challenges. Journal of General Physiology vol. 145 381-388 (2015).

37. La Rosa, G., Bonadonna, L., Lucentini, L., Kenmoe, S. \& Suffredini, E. Coronavirus in water environments: Occurrence, persistence and concentration methods - A scoping review. Water Research vol. 179115899 (2020).

38. Michael, P. R., Johnston, D. E. \& Moreno, W. A conversion guide: Solar irradiance and lux illuminance. J. Meas. Eng. 8, 153-166 (2020).

39. Tyagi, A. \& Penzkofer, A. Absorption and emission spectroscopic characterization of lumichrome in aqueous solutions. Photochem. Photobiol. 87, 524-533 (2011).

40. Eckl, D. B. et al. Interplay of phosphate and carbonate ions with flavin photosensitizers in photodynamic inactivation of bacteria. PLoS One 16, e0253212 (2021).

41. Malik, Z., Ladan, H. \& Nitzan, Y. Photodynamic inactivation of Gram-negative bacteria: Problems and possible solutions. J. Photochem. Photobiol. B Biol. 14, 262-266 (1992).

42. EMA. Committee for Medicinal Products for Human use (CHMP) Committee for Medicinal Products for Veterinary use (CVMP) Guideline on the sterilisation of the medicinal product, active substance, excipient and primary container. www.ema.europa.eu (2019).

43. Andreev, K. et al. Guanidino groups greatly enhance the action of antimicrobial peptidomimetics against bacterial cytoplasmic membranes. Biochim. Biophys. Acta - Biomembr. 1838, 2492-2502 (2014).

44. Kasai, H. \& Yamaizumi, Z. Photosensitized Formation of 7,8-Dihydro-8-oxo-2'-deoxyguanosine (8-hydroxy-2'deoxyguanosine) in DNA by Riboflavin: A Non Singlet Oxygen-Mediated Reaction. Journal of the American Chemical Society vol. 114 9692-9694 (1992).

45. Ito, K., Inoue, S., Yamamoto, K. \& Kawanishi, S. 8-Hydroxydeoxyguanosine formation at the 5' site of 5'-GG-3' sequences in double-stranded DNA by UV radiation with riboflavin. J. Biol. Chem. 268, 13221-13227 (1993).

46. Zhou, Z. Y. \& Bi, X. X. Experimental studies on the inactivation of HBV in blood via riboflavin photochemical treatment. Exp. Ther. Med. 13, 222-224 (2017).

47. Zhou, Z. Y. \& Bi, X. X. Evaluation of the inactivation effect of riboflavin photochemical method on duck hepatitis B virus. Exp. Ther. Med. 15, 751-754 (2018).

48. European Agency for the Evaluation of Medicinal Products. Note for Guidance on Virus Validation Studies: the Design , Contribution and Interpretation of Studies Validating the Inactivation and Removal of Viruses Cpmp/Bwp/268/95. Committee for Proprietary Medicinal Products (CPMP) vol. 0 http://www.ema.europa.eu/docs/en_GB/document_library/Scientific_guideline/2009/09/WC500003684.pdf (1996).

49. Malenovska, H. Virus quantitation by transmission electron microscopy, TCID50, and the role of timing virus harvesting: A case study of three animal viruses. J. Virol. Methods 191, 136-140 (2013).

50. Burgstaller, P. \& Famulok, M. Flavin-dependent photocleavage of RNA at G.U base pairs. J. Am. Chem. Soc. 119, 1137-1138 (1997).

51. Liu, Y. et al. Photosensitized damage to telomere overhang and telomerase RNA by riboflavin. Prog. Nat. Sci. 18, 401-404 (2008).

52. Zhao, Y. et al. Sequence-specific RNA photocleavage by single-stranded DNA in presence of riboflavin. Sci. Rep. 5, (2015). 
53. Kappus, H. Oxidative stress in chemical toxicity. Arch. Toxicol. 60, 144-149 (1987).

54. Reed, L. J. \& Muench, H. A simple method of estimating fifty per cent endpoints. Am. J. Epidemiol. 27, 493497 (1938).

55. Leibowitz, J., Kaufman, G. \& Liu, P. Coronaviruses: Propagation, quantification, storage, and construction of recombinant mouse hepatitis virus. Curr. Protoc. Microbiol. 21, 15E.1.1-15E.1.46 (2011).

\section{Figures}

Type I

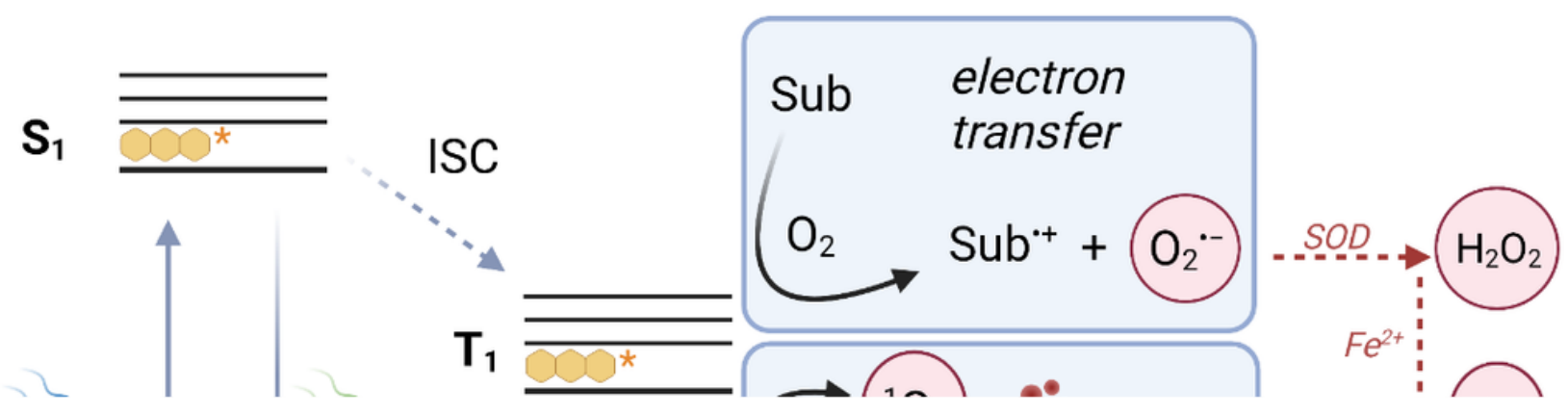

\section{Figure 1}

Mechanism of photosensitised reactive oxygen species (ROS) formation that enables inactivation of pathogens. $\mathrm{ISC}=$ intersystem crossing $\mathrm{Sub}=$ substrate, $\mathrm{SOD}=$ superoxide dismutase. 
a

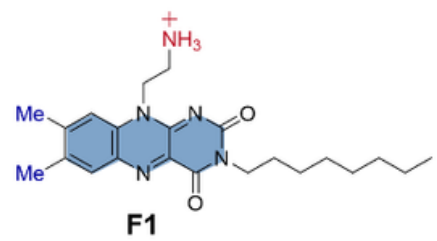

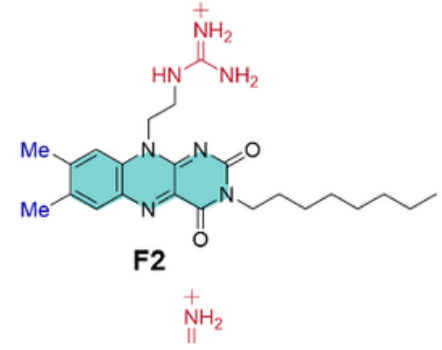

b

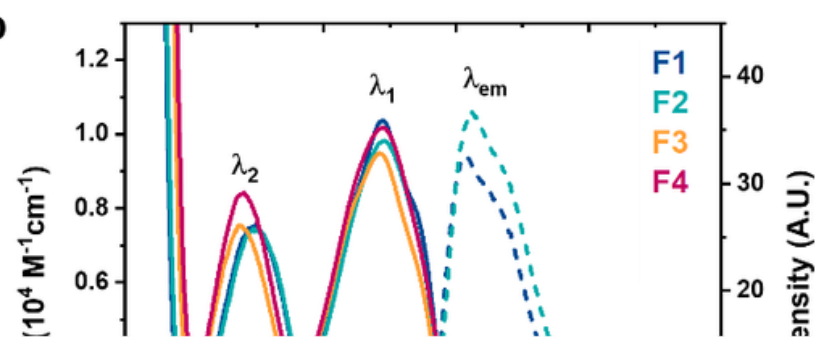

Figure 2

The structures and photophysical spectra of F1-4. a) Structures of flavins F1-4 (counteranions = TFA'), b) UV-Vis absorption (bold) and emission (dashed) spectra of F1-4 in DMSO.
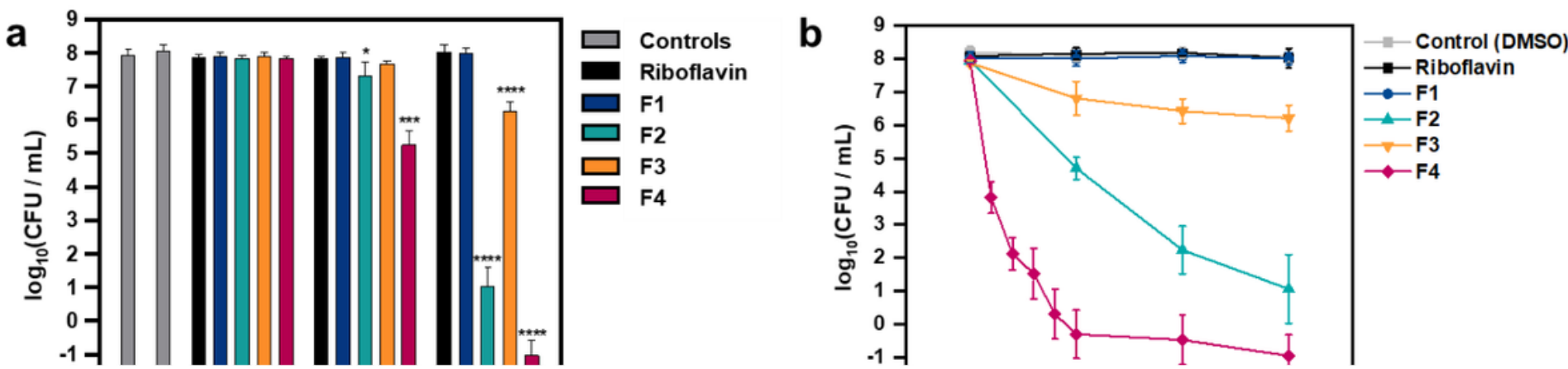

Figure 3

Photodynamic inactivation of $E$. coli. a) Photodynamic inactivation of E. coliBL21(DE3) incubated with various concentrations of flavins irradiated with white LED light $\left(1 \times 10^{5} \mathrm{Ix}, 35 \mathrm{~mW} / \mathrm{cm}^{2}\right)$ for $15 \mathrm{~min}$ in PBS containing 
0.5\% DMSO. Data are expressed as the mean \pm SD of three biological replicates. Significance levels are defined compared to PBS control as the following: * for $p \leq 0.05$, ** for $p \leq 0.01$, *** for $p<0.001$, and $* * \star *$ for $p<0.0001$. b) Time course of $E$. coli inactivation incubated with $100 \mu \mathrm{M}$ of flavin irradiated with white LED light $\left(1 \times 10^{5}\right.$ Ix, 35 $\mathrm{mW} / \mathrm{cm}^{2}$ ) for $15 \mathrm{~min}$ in PBS containing 0.5\% DMSO. Data are expressed as the mean \pm RE of three biological replicates. c) SIM images depicting the localisation of flavins in E. coliafter incubation with $100 \mu \mathrm{M}$ flavin. d) Agarose gel electrophoresis of pDNA (pUC18) following incubation with $10 \mu \mathrm{M}$ flavin irradiated with white LED light $\left(1 \times 10^{5} \mathrm{Ix}, 35 \mathrm{~mW} / \mathrm{cm}^{2}\right)$ for $15 \mathrm{~min}$ in PBS containing $0.1 \%$ DMSO. Rbf = Riboflavin.
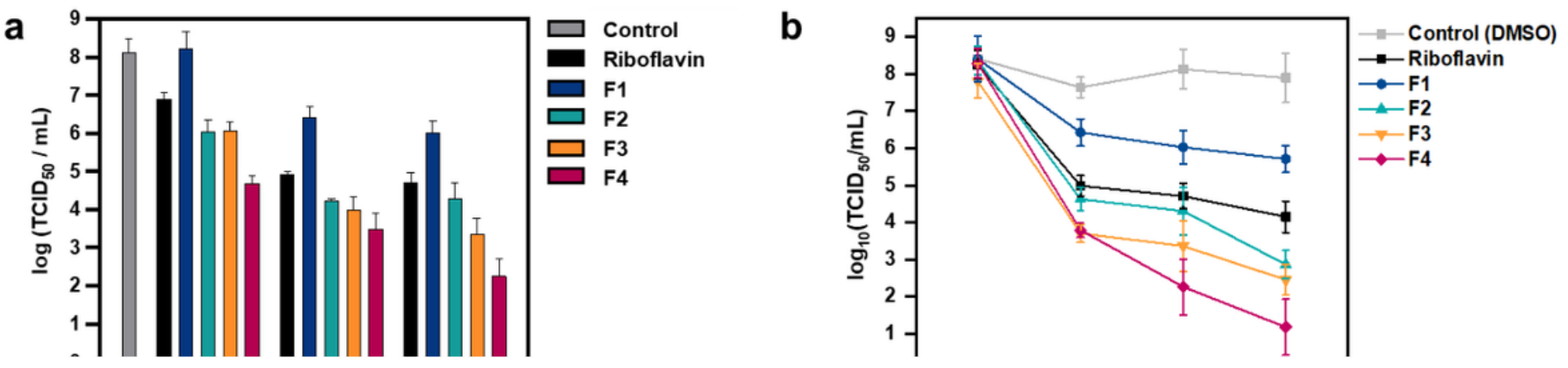

\section{Figure 4}

Photodynamic inactivation of murine hepatitis virus (MHV-A59). a) Photodynamic inactivation of murine hepatitis virus A59 strain (MHV-A59) incubated with various concentrations of flavins irradiated with white LED light $\left(1 \times 10^{5} \mathrm{Ix}, 35 \mathrm{~mW} / \mathrm{cm}^{2}\right)$ for $10 \mathrm{~min}$ in PBS containing $0.1 \%$ DMSO. Data are expressed as the mean \pm SD of three biological replicates. b) Time course of MHV-A59 inactivation incubated with $10 \mu \mathrm{M}$ of flavin irradiated with white LED light $\left(1 \times 10^{5} \mathrm{Ix}, 35 \mathrm{~mW} / \mathrm{cm}^{2}\right)$ for $15 \mathrm{~min}$ in PBS containing $0.1 \%$ DMSO. Data are expressed as the mean \pm RE of three biological replicates. c) Photodynamic inactivation of MHV-A59 incubated with $10 \mu \mathrm{M}$ of flavin compound irradiated with white LED light $\left(1 \times 10^{5} \mathrm{Ix}, 35 \mathrm{~mW} / \mathrm{cm}^{2}\right)$ or incubated in the dark for $15 \mathrm{~min}$ in PBS containing $0.1 \%$ DMSO. Data are expressed as the mean \pm SD of three biological replicates. d) Agarose gel electrophoresis of ssRNA following incubation with $10 \mu \mathrm{M}$ flavin irradiated with white LED light $\left(1 \times 10^{5} \mathrm{Ix}, 35\right.$ $\mathrm{mW} / \mathrm{cm}^{2}$ ) for $15 \mathrm{~min}$ in PBS containing $0.1 \%$ DMSO. Rbf = Riboflavin. 

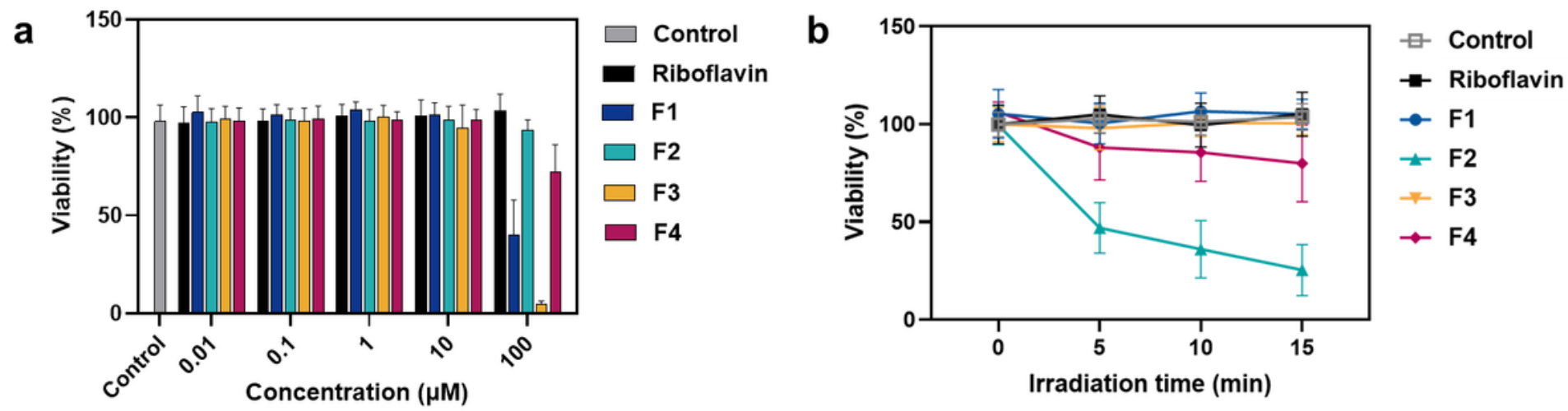

Figure 5

In vitro toxicity of F1-4 towards human cells. a) In vitro cytotoxicity effect of F1-4 and riboflavin on WI-38 cells after $24 \mathrm{~h}$ incubation determined by MTS assay. Data are expressed as the mean \pm SD of three biological replicates. b) Light-induced toxicity of WI-38 cells treated with F1-F4 and riboflavin (10 $\mu \mathrm{M})$ irradiated for 0-15 min with $1 \times 10^{5} \mathrm{I} x$ white LED light. Data are expressed as the mean \pm SD of three biological replicates. Control $=$ $0.1 \%$ DMSO in PBS.

\section{Supplementary Files}

This is a list of supplementary files associated with this preprint. Click to download.

- FlavinPDIESIV2.pdf 